\title{
THEORETICAL ASPECTS OF SMALL-SCALE PHOTOSPHERIC MAGNETIC FIELDS
}

\author{
M. SCHÜSSLER ${ }^{1}$ \\ Universitäts-Sternwarte \\ Geismarlandstr. 11 \\ D-9400 Göttingen \\ Federal Republic of Germany
}

\begin{abstract}
The state of theoretical description of small-scale concentrated magnetic fields in the solar photosphere (excluding oscillations and wave propagation) is reviewed with emphasis on work done since 1982 . The processes which probably lead to the formation of strong fields (flux expulsion, convective collapse) are discussed in some detail and the present understanding of the subsequent (quasi-)equilibrium state is summarized. We consider in particular the magnetic and thermal structure of the basic magnetic flux concentrations (magnetic elements) and stress the importance of radiative transfer effects, e.g. the horizontal heat exchange with the surroundings and the effect of radiation from the hot bottom and walls on the upper layers. Velocity fields within and around magnetic flux concentrations are discussed with emphasis on shift and asymmetry of the observed Stokes $V$-profiles which have recently been understood in terms of a downflow in the immediate vicinity outside magnetic structures. Reconnection and instabilities are considered as possible destruction processes for magnetic elements.
\end{abstract}

\section{Introduction}

Most of the observable magnetic flux permeating the solar photosphere is organized in a hierarchy of structures which have a magnetic pressure comparable to the gas pressure in their apparently non-magnetic environment. Detailed analysis of spatially unresolved spectra (reviewed by Solanki in these proceedings, see also Stenflo, 1989) indicates the existence of a basic structure, the magnetic element, with a magnetic flux of a few times $10^{17} \mathrm{mx}$, a flux density of about 2000 Gauss and a diameter of less than $200 \mathrm{~km}$ (both at continuum optical depth unity within the magnetic structure). Magnetic elements comprise most of the flux in the magnetic network outside active regions and in plage areas. Larger structures like sunspots or pores are formed in the course of the eruption of new active regions. After the initial phases of magnetic flux emergence they sooner or later fragment into magnetic elements. The ubiquity of magnetic elements and the remarkable fact that they all basically share the same properties place these structures in the focus of observational and theoretical interest, even more so since they are suspected to play a crucial part in the solution of the long-standing problems of chromospheric/coronal heating and the acceleration of the fast solar wind. Apart from the implication for solar and stellar physics, small-scale

1 Permanent address: Kiepenheuer-Institut für Sonnenphysik, Schöneckstr. 6, D-7800 Freiburg, Federal Republic of Germany 
magnetic flux concentrations in the solar (sub-)photosphere can be seen as an example for the formation of dissipative structures in systems far from thermal equilibrium.

Magnetic elements are too small to be individually studied in detail with presently existing spectroscopic instrumentation. Their tentative identification with small bright structures in the continuum and in spectral lines (Dunn and Zirker, 1973; Mehltretter, 1974; cf. review by Muller in these proceedings) has been supported by the analysis of spectral lines profiles (Schüssler and Solanki, 1988) and numerical model calculations (Spruit, 1976; Grossmann-Doerth et al., 1989a). Recently, it has been demonstrated by high-resolution magnetograms and filtergrams (Title et al., 1989) that magnetic structures coincide with bright features in the network while in plage regions the relation between brightness and magnetic structures seems to be more complicated. This is presumably due to the tendency of magnetic elements to collect into clusters in regions of large average magnetic filling factor (Knölker and Schüssler, 1988) where they strongly influence the granular motions and the convective energy transport.

The remarkable progress in our understanding of small-scale magnetic fields in the solar photosphere in spite of the resolution problem was made possible by the ingeneous use of indirect spectroscopic methods, the development of sophisticated instruments, most notably the Kitt Peak Fourier transform spectrograph/polarimeter (FTS), the theoretical study of basic physical processes in flux concentrations and magnetoconvection using simplified models, and the advent of comprehensive numerical simulations of MHD and radiative transfer.

Theoretical aspects of small-scale photospheric magnetic fields have been reviewed earlier by Meyer (1976), Weiss (1977), Parker (1979), Priest (1982), Spruit (1983), Spruit and Roberts (1983), Nordlund (1984,1985b,1986), Thomas (1985), Schüssler $(1986,1987 a)$, and Spruit et al. (1989). This review concentrates on the developments which took place after the IAU-Symposium No. 102 in Zürich (Stenflo, 1983). A number of reviews in these proceedings is related to magnetic elements, namely those of Müller, Solanki and Title (observational aspects), Nordlund (interaction with convection), and Ryutova (waves and oscillations). In order to limit overlapping with the latter two contributions, magnetoconvection and the theory of waves in fluxtubes will not be discussed here. We shall focus our attention on the present state of theoretical understanding of the formation (Ch.2) and destruction ( $\mathrm{Ch} .4$ ) processes of magnetic elements and to the properties of their quasiequilibrium state (Ch. 3 ).

\section{Formation}

Magnetic flux which is observed in the solar photosphere most probably has its origin in dynamo processes operating near the bottom of the convection zone from where it rises to the surface due to the combined effects of buoyancy and convective flows. Since the average field strength in an emerging flux region is rather high, a pre-eruption field strength of at least a few hundred Gauss in the uppermost layers of the convection zone must be assumed (Zwaan, 1978). The erupting flux transforms into strong flux concentrations in a matter of minutes since there is no evidence that the field strength in newly erupted active region is smaller. After the initial flux eruption we expect a dynamical state : Flux concentrations are formed, temporarily attain an equilibrium state and dissolve again while the magnetic flux is constantly moved around the changing pattern of granulation and supergranulation. On the other hand, at any instant from the beginning of the life of an active region to the 
dispersal of its flux in the network more than $90 \%$ of the magnetic flux which is observable through the analysis of circular polarization in spectral lines (see Stenflo, 1987, on the possible existence of a "turbulent" field with mixed polarity on very small scales) is in the form of magnetic elements with large field strength. Consequently, the formation and destruction processes must have a much shorter timescale than the lifetime of magnetic elements in equilibrium.

The remarkable result that all magnetic elements irrespective of being located in network or in active regions have similar thermodynamic and magnetohydrodynamic properties (e.g. Stenflo and Harvey, 1985; Zayer et al., 1989) which vary only weakly with increasing number density of flux concentrations (Solanki and Stenflo, 1984) indicates that they are formed by essentially the same process and reach a (quasi-)equilibrium which is determined by the local properties of the plasma within and around them. There are two mechanism which are held responsible for the concentration of magnetic flux into structures with large flux density: Flux expulsion and convective collapse. Although both processes are related we discuss them in somewhat artificial separation in order to emphasize the basic effects.

Convective motions in an electrically well-conducting plasma concentrate magnetic flux into structures with a local flux density much larger than its average value. This flux expulsion process (Parker, 1963; Weiss, 1966; Galloway and Weiss, 1981; Weiss, 1981a,b; Hurlburt et al., 1984; Hurlburt and Toomre, 1988) leads to a kind of "phase separation" between field-free convecting plasma and magnetic, almost stagnant regions. It has been suggested by Parker (1984) that in a stellar convection zone such a configuration is energetically favoured since it minimizes the interference of the magnetic field with the convective energy transport. In the case of the solar (sub-)photosphere, flux expulsion leads to a sweeping of magnetic flux into the intergranular downflow regions as demonstrated by high-resolution observations (Title et al., 1987) and numerical simulations (Nordlund 1983, 1986). The expulsion process works in essentially the same way for the vertical component of the vorticity as can be shown using the well-known formal identity of the equations describing the time evolution of vertical vorticity and magnetic field in the kinematical limit. This has the consequence that both magnetic flux and vertical vorticity are concentrated into the narrow downflow regions of granular convection (cf. Nordlund, 1985a,b) such that the magnetic flux concentrations become surrounded by rapidly rotating, descending whirl flows.

The back reaction of the magnetic field on the flow via the Lorentz force limits the flux density which can be achieved by flux expulsion to a value which is roughly given by the equipartition of magnetic and kinetic energy density. This limit may be modified by the effects of diffusion and compressibility (cf. Proctor and Weiss, 1982). For the case of the solar (sub-)photosphere, however, the equipartition limit (a few hundred Gauss) is rendered irrelevant by thermal effects. Since the horizontal flows of granular convection are responsible for both sweeping the magnetic field to the downflow regions and for carrying heat to those regions, the retardation of the flows by the growing magnetic field leads to a cooling of the magnetic region since the the radiative losses can no longer be balanced by the throttled horizontal flow. This cooling effect causes an increase of the magnetic field since the gas pressure in the magnetic region becomes smaller and it accelerates the downflow which gives rise to the superadiabatic effect (Parker, 1978): An adiabatic downflow in a magnetic flux tube which is thermally isolated from its surroundings leads to a cooling of the interior with respect to the superadiabatically stratified surroundings and a partial evacuation of the the upper layers ensues. Pressure equilibrium with the surrounding gas 
is maintained by a contraction of the flux tube which increases the magnetic pressure. In this way, the magnetic field can be locally intensified to values which are only limited by the confining pressure of the external gas.

It has been shown by a number of authors (Webb and Roberts, 1978; Spruit and Zweibel, 1979; Unno and Ando, 1979) that the superadiabatic effect in the case of a flux tube which is in magnetostatic and temperature equilibrium with its environment drives a convective instability in the form of a monotonically increasing up- or downflow. Consequently, the initial downflow due to the radiative cooling will be enhanced by this effect leading to an even stronger amplification of the magnetic field, a process which is often referred to as convective collapse.

While this convective instability of a flux tube with a weak magnetic field is theoretically well established, the results for strong fields and for the nonlinear evolution of the convective collapse give no unique picture. The claim of Spruit and Zweibel (1979) and Spruit (1979) that flux tubes with a strong enough magnetic field become stable with respect to convective collapse has been critized by Nordlund (1984) who argued that this result depends on the choice of boundary conditions: If the displacement of matter (or the fluid velocity along the tube) is not constrained to vanish at two fictitious endpoints of the tube, any adiabatic downward displacement leads to a state of lower energy and there is no stable equilibrium, irrespective of the field strength. This argument is supported by the linear results of Webb and Roberts (1978) who showed that the location of the lower (closed) boundary significantly influences the linear stability in a way that the stabilizing effect of the magnetic field decreases more and more as the location of the lower boundary is shifted deeper and deeper.

This dependence on the boundary conditions explains the discrepancy between the results of nonlinear simulations by Hasan (1983) and Venkatakrishnan (1983) and those performed later by Hasan (1984). In the first two papers constant internal gas pressure was assumed at the boundaries which thus were effectively "open". In these cases the convective instability evolved into a state of permanent downflow with high velocity, nearly independent of the strength of the initial field. In his subsequent paper, Hasan (1984) used closed boundaries, i.e. vanishing velocity at the end points. Now the instability was suppressed for strong enough magnetic field (in agreement with the linear results using the same boundary conditions) and the unstable configurations evolved into a state of stationary adiabatic oscillation.

With the exception of very special upper boundary conditions (e.g. Unno and Ando, 1979), the crucial point is the choice of the lower boundary condition (cf. Webb and Roberts, 1978; Hasan, 1986). A downflow leads to a gas pressure enhancement near a closed boundary and a local expansion of the flux tube in order to maintain equilibrium with the external gas pressure. For a weak field, this expansion is significant since only a moderate increase of the internal gas pressure can be balanced by a decrease of the magnetic field. Consequently, the vertical restoring force on the downflow due to the pressure enhancement is weak and the expansion of the tube provides space for the matter carried by the nearly unimpeded downflow. With a strong magnetic field, on the other hand, pressure balance with the exterior is readjusted by only a slight expansion and the internal gas pressure enhancement is fully available as restoring force in the vertical direction. However, if the flow is not constrained to vanish at the boundary or if the internal pressure is assumed to be constant there, the restoring force is much less effective and a strong magnetic field is not able to suppress the instability. 
The question arises as to which boundary conditions should be used in the case of solar photospheric magnetic flux concentrations. Strictly speaking, a vanishing of the fluid displacement can reasonably be assumed only in a convectively stable stratification, i.e. if the bottom of the tube is placed below the convection zone. This has effectively been done by Webb and Roberts (1978, Ch. 6.2) by forcing the velocity perturbation to vanish at infinity and also by Spruit and Zweibel (1979) who put the boundary at the bottom of the convection zone. While Webb and Roberts (1978) found for a model with a constant temperature gradient that a strong field can only stabilize a small range of superadiabatic temperature gradients, Spruit and Zweibel (1979) showed that for their boundary conditions and a realistic convection zone a flux tube is stable if $\beta=8 \pi p / B^{2}<1.51$. Both calculations assume an equlibrium state with temperature in the flux tube being equal to the external temperature, i.e. a depth-independent value of $\beta$. The reason for the differing results lies in the small superadiabaticity of the deeper layers of a realistic convection zone while Webb and Roberts (1978) assume a constant superadiabaticity. However, the applicability of these linear results for strong fields to the real Sun is questionable for a number of reasons:

- The assumption of adiabatic changes is quite unrealistic because of the strong effects of radiation on the energy balance of the surface layers.

- Taking a depth-independent $\beta$ implies unrealistically large values of the field strength in the deep layers: $3 \cdot 10^{8}$ Gauss at the bottom of the convection zone for $\beta=1.5$.

- It is by no means obvious that the observed magnetic elements should maintain their identity as single flux tubes in deeper layers and the assumption of a vertical tube becomes inadequate at moderate depths of a few $10^{3} \mathrm{~km}$ : Small flux concentrations become passive with respect to the convective flows due to the strong increase in density and will be severely distorted and fragmented (Schüssler, 1984a; 1987b).

In view of the observational results which imply that most of the magnetic flux at any given instant of time is in the form of magnetic elements approximately in hydrostatic equlibrium without a significant downflow (Stenflo and Harvey, 1985; Solanki, 1986) all of which have similar thermodynamic and magnetic properties, we conclude that this state cannot be determined by conditions deep within the convection zone but rather is controlled by the local conditions in or near the observable layers. In summary, although the linear results demonstrate the existence of the convective collapse mechanism they do not seem to be particularly relevant for the questions whether a stable state is reached by the instability and, if yes, which are its properties. The dynamics of the nonlinear evolution of the instability and its non-adiabatic character due to radiative effects have to be taken into account in order to quantitatively predict the result of a convective collapse.

The configuration resulting from the convective collapse must not necessarily be globally stable in order to represent a local quasi-equilibrium in the surface layers as implied by observations. The radiative cooling effect and the large superadiabaticity are restricted to the uppermost layers of the convection zone and the resulting unstable downflow will be localized in this region. The deeper layers are only slightly superadiabatic and thus almost neutrally stable but represent a large inertia because of the drastic increase of density with depth. We should therefore expect that the collapsing upper layers are stopped and reflected similar to a body colliding with another body of much larger mass at rest which is set into only very slow motion. It is presently unclear how strong the following upflow is. Nordlund's $(1983,1986)$ simulations do not show an upflow but their poor spatial resolution and strong numerical diffusion on the scale of the flux concentrations couple the 
magnetic regions artificially to the dynamics of the external downflow. The simulations of Hasan $(1984,1985)$ and Venkatakrishnan $(1983,1985)$ exhibit an upflow and a subsequent oscillation but they do not incorporate a precise treatment of the important effects of vertical radiative energy losses which are largely enhanced by the strong temperature dependence of the continuum opacity. If the upflow is strong enough it could possibly drive a spicule through the formation of shocks (cf. Suematsu et al., 1982; Hollweg, 1982; Sterling and Hollweg, 1988). However, it is well possible that the matter has lost so much energy through radiative losses that the upflow is weak or even non-existent. Eventually, this issue has to be settled by observation where the only indication of a convective collapse has been provided by Wiehr (1985; see, however, Solanki and Stenflo, 1986). It would be a major achievement if spectroscopic observations with high spatial resolution could follow the convective collapse of a single magnetic flux concentration.

Whichever is the detailed dynamic evolution of the convective collapse, in the uppermost layers of the convection zone it leads to a state near hydrostatic equlibrium. Due to the strong radiative losses during the collapse phase the gas in the flux concentration is now significantly cooler than the surrounding medium at equal depth. This temperature reduction is well capable of stabilizing the equilibrium of the upper layers with respect to further convective collapse (see Webb and Roberts, 1978) independent of the choice of particular boundary conditions in the linear stability analysis. Thus it is the non-adiabaticity of the collapse due to the radiative energy losses which may well be responsible for the establishment of a locally stable configuration while the increase in magnetic field strength only is of minor importance.

The thermal isolation with respect to convection is counteracted by radiation if the magnetic structure is so small that its horizontal optical depth becomes of order unity. Consequently, very small structures will always be kept at the temperature of the surrounding gas and therefore cannot undergo a convective collapse (Schüssler, 1986; Venkatakrishnan, 1986). Hasan (1986) has shown for a realistic solar atmosphere that the critical value of $\beta$ for the onset of monotonic convective instability increases rapidly for decreasing diameter of the flux tube if lateral radiative energy exchange is taken into account.

It remains an open question whether magnetic elements are susceptible to overstability caused by horizontal radiative transfer (Roberts, 1976; Spruit, 1979; Hasan, 1985, 1986; Venkatakrishnan, 1985; Massaglia et al., 1989) if the energy losses by vertical radiative transfer are consistently taken into account. A proper treatment of radiation far beyond the limits of the diffusion equation or "Newton's law of cooling" and an adequate level of spatial resolution in numerical simulations is necessary in order to decide whether overstable oscillations are excited in a realistic model of a magnetic element. Even a crude inclusion of vertical radiative transfer (Venkatakrishnan, 1985) or the step from the radiative diffusion/relaxation time approach to the Eddington approximation (Massaglia et al., 1989; Hasan, 1989) led to drastic changes especially for the interesting case of a flux tube with a horizontal optical depth around unity. 


\section{Equilibrium}

Observational results indicate that photospheric magnetic flux concentrations reach an equilibrium state whose properties are similar for most small-scale flux concentrations (Zayer et al., 1989) and depend only weakly on the filling factor, i.e. the fraction of the area covered with magnetic elements (Stenflo and Harvey, 1985; Solanki and Stenflo, 1984). The upper limit of $250 \mathrm{~m} \cdot \mathrm{s}^{-1}$ for an average flow within the magnetic structures determined from the absolute shift of the Stokes $V$-profile zero crossings of spectral lines in spatially and temporarily unresolved FTS spectra (Stenflo and Harvey, 1985; Solanki, 1986) indicates that the structures are approximately in (magneto-)hydrostatic equlibrium. This upper limit also excludes efficient vertical convective energy transport, for instance by large-amplitude overstable oscillations, since this would also lead to a significant average shift due to a correlation between intensity and velocity (e.g. Hasan, 1985).

These results justify the theoretical working hypothesis that, except for the formation and destruction phases, the basic properties of photospheric magnetic flux concentrations may be represented by prototype flux tubes or flux slabs in static or stationary equilibrium embedded in a non-magnetic environment. The theoretical objective is to describe this state self-consistently including force balance and dynamics, energy transport by radiation and flows, and interaction with the environment. Ultimately, this task calls for a comprehensive 3D time-dependent numerical simulation. However, this cannot by achieved with the computational facilities available at present or in the near future. For example, the simulations by Nordlund $(1983,1986)$ have a spatial resolution of about $250 \mathrm{~km}$ in the horizontal direction while a value of a few $\mathrm{km}$ is needed to resolve the boundary layer between a flux concentration and its surroundings which is crucial for a correct description of the energy balance. Consequently, the available 3D simulations may describe the average motion of an ensemble of magnetic elements in a time-dependent granular velocity field but they give no information on the dynamics and the properties of individual flux concentrations. So it is necessary to consider models restricted to one or two spatial dimensions which allow a much better spatial resolution.

Some guidance as to the assumptions going into these models can be taken from simple considerations. For example, the strong buoyancy force of a magnetic element keeps it essentially vertical in the (sub-)photospheric layers (Schüssler, 1986) such that the model of a vertical flux tube with a straight axis seems reasonable unless its diameter is much smaller than the scale height. Also the assumption of a thin boundary (current sheet) between the flux concentration and its environment is supported by estimates of the widths of resistive and viscous boundary layers (Schüssler, 1986). The cross-field flows due to finite resistivity are less than $10 \mathrm{~m} \cdot \mathrm{s}^{-1}$ for the photospheric regions while the drift velocities of neutral atoms is a few $\mathrm{cm} \cdot \mathrm{s}^{-1}$ at maximum (Hasan and Schüssler, 1985). Thus the effects of finite resistivity and incomplete ionization are irrelevant and the approximation of ideal magnetohydrodynamics is well justified for the photospheric layers.

\subsection{MAGNETIC FIELD}

In order to describe the structure of the magnetic field in a photospheric flux concentration a number of approaches has been used. Static models based on the approximation of slender fluxtubes have been presented by Ferrari et al. (1985) and Hasan (1988). Higher orders in the radial expansion procedure which to zeroth order gives the slender fluxtube approximation (cf. Roberts and Webb, 1978; Ferriz-Mas and Schüssler, 1989) and allow to include twisted 
fields have been considered by Wilson (1977), Browning and Priest (1983) and Pneuman et al. (1986). The similarity approach was used by Osherovich et al. (1983) and Solov'ev (1984). 2D models of potential fields have been presented by Spruit $(1976,1977)$, Simon et al. (1983) and by Schmidt and Wegmann (1983, see also Jahn, these proceedings) who solved the free boundary problem consistently . Full 2D magnetostatic models with internal currents and current sheets have been published by Pizzo (1986), Steiner et al. (1986) and recently by Steiner and Pizzo (1989).

Time dependence is included in a number of approaches which aim either at describing dynamical processes (flows, waves, oscillations, shocks) or try to model the evolution of the flux concentration to a stationary state self-consistently. In this connection slender flux tubes have been assumed, among others, by Unno and Ribes (1979), Hollweg (1982), Hasan (1984, 1985), Venkatakrishnan (1983, 1984), Herbold et al. (1985), Ribes et al. (1985), Hasan and Schüssler (1985), Ferriz-Mas and Moreno-Insertis (1987), Ferriz-Mas (1988), Thomas (1988), Montesinos and Thomas (1989), and Degenhardt (1989). Higher orders of the radial expansion have been considered by Ferriz-Mas et al. (1989) and Anton (1989). Simulations in 2D slab geometry have been performed by Deinzer et al. (1984a,b), Knölker et al. (1988), Knölker and Schüssler (1988) and Grossmann-Doerth et al.(1989a). The path followed by slender flux tubes in prescribed cellular velocity fields meant to represent supergranular and granular flows has been investigated in a time-dependent calculation by Meyer et al. (1979) and Schmidt et al. (1985).

The height-dependence and the horizontal constancy of the magnetic field given by the slender flux tube approximation are in good agreement with results derived from FTS spectra in the visible and in the infrared (Zayer et al., 1989), a result which is further supported by comparison with 2D models (Knölker et al., 1988). Steiner and Pizzo (1989) have shown that an unrealistically large amount of heating or cooling in the photospheric layers of a flux tube would be necessary in order to significantly influence its shape. The effect of a twisted field on the equilibrium and shape of a flux tube has been investigated by Steiner et al. (1986) who found that magnetostatic equilibrium cannot be achieved if the azimuthal field component exceeds a critical value of about a third of the axial field strength at the base of the model. For statically allowed values of the twist the shape of the flux tube and the height where it merges with the neighbouring flux tubes for a given filling factor is not strongly affected by twisting the field (see also Pneuman et al., 1986).

\subsection{THERMAL STRUCTURE}

A variety of effects influences the energetics and the temperature structure of a photospheric flux concentration:

- Advection of heat by flow fields in the environment,

- lateral exchange of energy with the surroundings by radiative transport,

- reduction/suppression of convective energy transport by a strong magnetic field,

- vertical radiative loss, anisotropic radiation field.

A proper treatment of the radiative energy transport is crucial for quantitative modelling of the thermodynamics of magnetic elements since radiation largely determines the energy budget. Besides models which deal with specific aspects of the radiative energy transport (e.g. the lateral energy exchange by a relaxation time appoach) there is a number of investigations which attempt to include radiation more comprehensively. The comparatively 
simplest approach is to take a slender flux tube which is assumed to be optically thin, i.e. has a diameter smaller than the photon mean free path. In this case the mean intensity is mainly determined by the external medium and the temperature is nearly constant in horizontal planes (Ferrari et al., 1985; Kalkofen et al., 1986). It turns out that the temperature as function of optical depth for such models is $1000-2000 \mathrm{~K}$ higher than that given by semiempirical models derived from Stokes $V$-profiles (Solanki, 1986; Keller, these proceedings). Consequently, optically thin flux tubes are not adequate to describe solar magnetic elements.

Other simplified approaches are the Eddington approximation for the case of a slender flux tube which has been used by Hasan (1988) and the 2D diffusion approximation (Spruit, 1976, 1977; Deinzer et al., 1984a,b). However, the anisotropy of the radiation field and the necessity to describe optically thick and optically thin regions equally well demands a full treatment of the radiative transfer by integration of the transport equation along many rays and angles. For the slab geometry and a grey atmosphere this has been incorporated in the time-dependent simulations of Grossmann-Doerth et al. (1989a) while Steiner (these proceedings) has included a non-grey radiative transport in magnetostatic models of cylindrical flux tubes. In a prescribed slab geometry resembling a flux concentration, Kalkofen et al. (1989) have determined a grey radiative equilibrium atmosphere which has qualitatively similar properties as the self-consistently determined models of Grossmann-Doerth et al. and Steiner.

The model calculations which incorporate a full radiative transfer revealed an important effect, i.e. the heating of the upper layers within the flux concentration by radiative illumination: The material in the region above optical depth unity of a partially evacuated magnetic element is bathed in the radiation from the hot bottom (with a temperature of more than $7000 \mathrm{~K}$ at $\left.\tau_{c}=1\right)$. It therefore reaches an equilibrium temperature which is larger than that of the gas at the same height in the quiet atmosphere which "sees" radiation from a layer of optical depth unity at a temperature of about $6400 \mathrm{~K}$. The result is the formation of a hot region with a temperature which is a few hundred degrees larger than that of the environment at equal geometrical depth. As an example, Fig. 1 shows the temperature distribution for the 2D slab model of Grossmann-Doerth et al. (1989a).

In the layers below $\tau_{c}=1$ the suppression of convective energy transport leads to a temperature deficit in the flux concentration (with respect to the nonmagnetic gas at the same depth far away from the flux concentration) which reaches a maximum of nearly 3000 $K$. The resulting horizontal temperature gradient drains energy from the external medium via a lateral radiative heat flux directed into the magnetic element. This inflow of heat balances the vertical radiative losses and limits the temperature deficit to a level which still leads to a temperature excess compared to the quiet Sun at equal optical depth for vertical incidence of the line of sight. These results are in qualitative agreement with semiempirical models determined from Stokes $V$-profiles (Solanki, 1986; Keller, these proceedings, see his Fig. 2) without the necessity to incorporate any mechanical heating. Still, potentially important effects have to be included in the comprehensive model calculations, namely a more detailed treatment of spectral lines, particularly those of $\mathrm{CO}$ which may have an influence on the temperature profiles in the layers above $\tau_{c} \approx 10^{-4}$ (see Ayres, these proceedings; Hasan and Kneer, 1986; Massaglia et al., 1988).

The lateral influx of heat by radiation, together with the partial evacuation of the flux concentration and the strong temperature dependence of the opacity has the consequence that the magnetic elements become brighter than the quiet atmosphere when observed in the continuum with high spatial resolution. At a wavelength of $5000 \AA$, the slab model of 
Grossmann-Doerth et al. (1989a) gives a value of about 1.6 of the intensity of the quiet Sun. Most of this excess intensity, however, is due to a redistribution of the heat flux: Energy has flown laterally into the flux concentration leading to a cooling of the exterior and the appearance of a darker region around the magnetic element. Only a small part of the heat flux disturbance propagates into the deeper layers where it is spread rapidly over the whole convection zone. In this way, the magnetic elements can act as a "heat leak" (Spruit, 1977, 1982). The amount of the net excess heat flux is difficult to predict precisely with presently available model calculations since it depends on the treatment of the convective energy transport and also on boundary conditions. However, both observation (Foukal and Fowler, 1984; Hirayama et al., 1985) and simulations (Deinzer et al., 1984b; Knölker et al., 1988) indicate that the net excess flux is likely to be small such that most of the large flux excess within the magnetic elements is compensated by a deficit in the surrounding non-magnetic atmosphere. Even though the excess flux is small for an individual magnetic structure, magnetic elements clustered in plage and network regions may well contribute significantly to the observed solar irradiance variations (cf. Foukal and Lean, 1987).

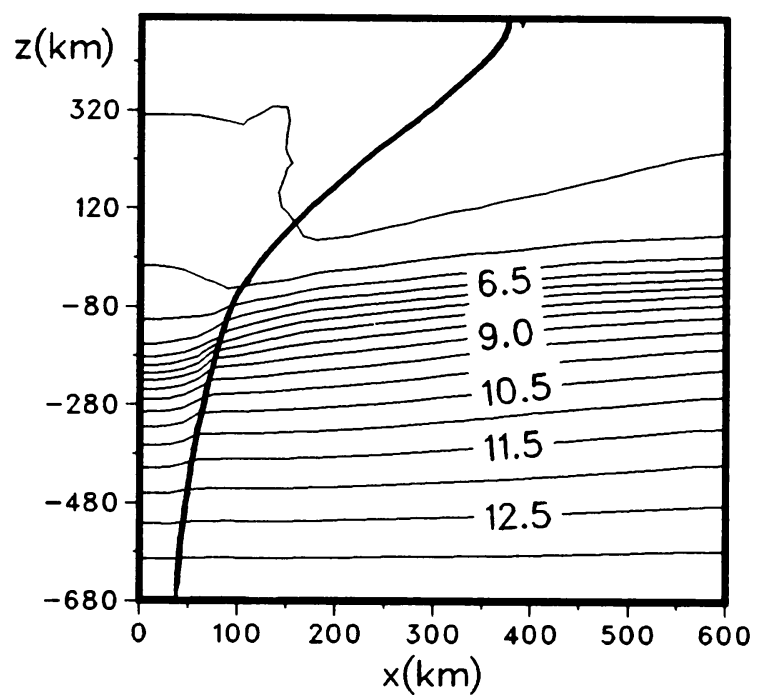

Fig. 1: Temperature distribution in the 2D slab model of Grossmann-Doerth et al. (1989a). The labels at the contour lines are given in units of $1000 \mathrm{~K}$. Only half of the symmetric structure is displayed and the boundary of the flux slab is indicated by the thick line. In the deeper layers the magnetic structure is cooler than the environment at the same height due to the suppression of convective energy transport while in the upper layers it is hotter than the surroundings because of radiative illumination from the hot bottom.

The center-to-limb variation of the intensity contrast has been discussed in some detail in an earlier review (Schüssler, 1987a; for a different point of view see Schatten et al., 1986). Three effects contribute to the variation of the intensity contrast for finite inclination of the line of sight:

- The hot bottom of the magnetic element is obscured for rather small inclinations leading to the disappearance of bright points (see Müller and Roudier, 1984; Müller et al., 1989); 
- the bright wall of the flux concentration becomes visible at large inclination angles and leads to a positive intensity contrast depending on the ratio between size and depth (Wilson depression) of the magnetic structure (Spruit, 1976);

- the hot upper regions of the magnetic elements overlap near the limb and lead to a sharp contrast increase (Steiner, these proceedings; see also Rogerson, 1961).

These effects are sufficient in order to understand the various observational results if spatial resolution, selection effects and the precise way of measurement are taken into account. High resolution observations near disc centre are mainly determined by the hot bottom of the magnetic elements, while the selection of individual bright "facular granules" (e.g. Muller, 1975 ) reveals the effect of the bright wall. The brightness evolution of individual faculae near the limb (Akimov et al., 1987), the observations of elevated faculae during eclipses (Akimov et al., 1982) and the measurements at the extreme limb (Chapman and Klabunde, 1982; Lawrence and Chapman, 1988) can be understood by the effect of overlapping hot regions.

\subsection{DYNAMICS}

The upper limit of $250 \mathrm{~m} \cdot \mathrm{s}^{-1}$ for the average velocities within magnetic elements determined from the observed $V$-profile zero-crossing shifts excludes strong systematic flows and largeamplitude overstable oscillations with intensity-velocity correlation. On the other hand, the large width of the $V$-profiles indicates the existence of "turbulence" with velocities of a few $\mathrm{km} \cdot \mathrm{s}^{-1}$ within magnetic elements. The nature of this velocity field is presently unknown but it is tempting to speculate that the various wave modes of a flux tube are excited by the interaction with convective flows and $p$-mode oscillations (Bogdan and Knölker, 1989; see also Roberts and Solanki, these proceedings) Flux tube oscillations and waves are discussed in more deatil by M. Ryutova elsewhere in these proceedings.

Another important indicator for dynamics associated with magnetic elements is the area and amplitude asymmetry of the observed Stokes $V$-profiles (Stenflo et al., 1984). Apart from atomic orientation (Kemp et al., 1984; Landi Degl'Innocenti, 1985) which seems inconsistent with the observed sign reversal of the asymmetry near the solar limb (Stenflo et al., 1987; Pantellini et al., 1988), a combination of magnetic field and velocity gradients along the line of sight appears to be the only reasonable explanation (Illing et al., 1975; Auer and Heasley, 1978; Sanchez-Almeida et al., 1988). However, flows within the magnetic structure in a physically realistic configuration (e.g. magnetic field decreasing with height) which reproduce the observed asymmetries lead to large shifts of the $V$-profile zero crossings which contradict the observations (Solanki and Pahlke, 1988).

Van Ballegooijen (1985) has suggested that an area and amplitude asymmetry of the $V$ profile may also be caused by a downflow outside but in the immediate vicinity of a static magnetic flux concentration: Since the magnetic field flares out with height, lines of sight at the periphery traverse static magnetic (upper part of the atmosphere) and downflowing nonmagnetic (lower part of the atmosphere) regions. It has been shown by Grossmann-Doerth et al. $(1988,1989 \mathrm{~b})$ that quite generally such a configuration leads to asymmetric $V$-profiles with unshifted zero crossings. Solanki (1989) was able to demonstrate that the observed $V$-profile area asymmetries of many spectral lines can be quantitatively reproduced in this way. Furthermore, since the downflows are feeded by horizontal flows directed towards the flux concentration this model at the same time accounts in a natural way for the sign reversal of the asymmetry shown by observations near the solar limb (Grossmann-Doerth et al., in preparation). Fig. 2 illustrates the geometry of the magnetic element and the surrounding flow field which leads to the formation of asymmetric $V$-profiles. 


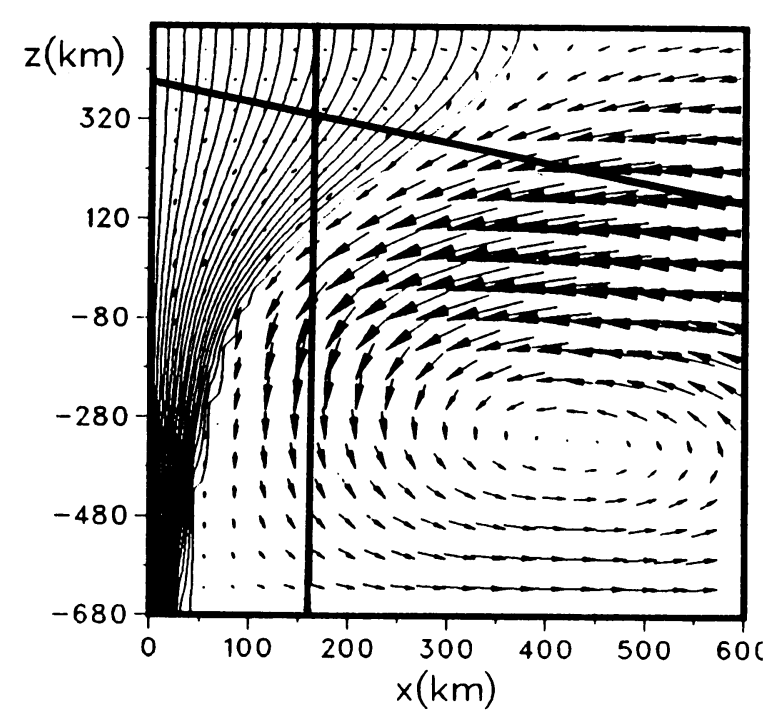

Fig. 2: Velocity and magnetic field structure of the slab model of Grossmann-Doerth et al. (1989a). Only half of the symmetric structure is displayed. While the interior of the magnetic element is almost static, a thermal circulation cell with a strong downflow (maximum velocity about $1.5 \mathrm{~km} \cdot \mathrm{s}^{-1}$ ) and large horizontal velocities (up to $2 \mathrm{~km} \cdot \mathrm{s}^{-1}$ ) has evolved in the non-magnetic environment. Two representative lines of sight are indicated which traverse static, magnetic regions and non-magnetic moving gas leading to asymmetric Stokes $V$-profiles. The vertical line "sees" a flow away from the observer while the inclined ray cuts a flow towards the observer such that the resulting asymmetries have different signs.

It seems as if the long-standing " $V$-profile dilemma" has found its resolution in the physically appealing concept of a magnetic element surrounded by a strong downflow in a cool environment. Photospheric magnetic flux is observed to be situated predominantly in the intergranular downflow regions (Title et al., 1987) which are also the site of the network bright points (Müller, 1983). The cooling effect of the magnetic elements on the surrounding gas supports and accelerates such a downflow (Deinzer et al., 1984b). The formerly enigmatic asymmetries thus constitute an important diagnostic tool for the structure of the flow field in the vicinity of magnetic elements. Additionally, the amplitude asymmetries may yield information about internal oscillations and waves (Solanki, 1989; Roberts and Solanki, these proceedings).

\section{Destruction}

The lifetime of individual magnetic elements is difficult to determine observationally. The simulations carried out by Nordlund $(1983,1986)$ show a continuous rearrangement of magnetic flux in the integranular lanes with a lifetime of the simulated magnetic structures (clusters of magnetic elements ?) determined by the timescale of the granular velocity field. However, due the low spatial resolution of his grid, the internal structure of the flux concentrations is unresolved and they are artificially coupled to the granular velocity field. 
The resolution of the 3D simulations has to be increased by at least an order of magnitude before they can contribute to the solution of this problem.

Muller (1983) found a mean lifetime of network bright points near disk center of about 20 minutes, but it is not clear whether this represents also the life span of the underlying magnetic structure. However, if the observed bright point represents the hot bottom layers of magnetic elements as indicated by the results discussed in $\mathrm{Ch} .3$ its fading signals a major change in its structure, possibly its dissolution.

A crude estimate of a minimum lifetime of a magnetic element in strong-field form can be derived from the lower limit of $90 \%$ for the fraction of magnetic flux (excluding the "turbulent" flux) in strong-field form (Howard and Stenflo, 1972) and the timescale of the convective collapse of 2 to 5 minutes (Hasan, 1985; Nordlund, 1986). Allowing for a quick destruction of the magnetic element by an instability (see below) within one minute we find a minimum lifetime between 30 and $60 \mathrm{~min}$ for the quasi-equilibrium state.

Which processes can possibly destruct magnetic elements ? In regions of mixed polarity, reconnection is important (Spruit et al., 1987). The result of reconnection of two opposite polarity magnetic elements depends on the location of the reconnection point: If it is below the surface an U-shaped loop forms which floats upwards due to magnetic buoyancy. It arrives there with a low field strength since the strong decrease of density with height and mass conservation leads to a significant expansion of the rising flux tube. Such a process possibly is a source of intrinsically weak magnetic field and might be related to the "intranetwork fields" (Martin et al., 1985; Livi et al., 1985). If reconnection takes place above the surface it forms a loop which can be drawn below the surface due to the action of magnetic tension forces if the footpoint separation is less than a few scale heights (Parker, 1979, Ch. 8). Both possibilities lead to quite different observational signatures (see discussion in Spruit et al., 1989).

An individual flux concentration can be destructed by dynamical processes, most efficiently by an instability. Besides the destabilizing influence of external flows related to the Kelvin-Helmholtz instability (e.g. Schüssler, 1979; Tsinganos, 1979), the interchange or fluting instability is most important (Parker, 1975). While pores and sunspots can be stabilized by gravity (Meyer et al., 1977), small flux concentrations are stable with respect to fluting if they are surrounded by a strong whirl flow (Schüssler, 1984b). Intermediate size structures cannot be stabilized by either effect which gives an upper limit for the size of magnetic elements depending on the maximum azimuthal velocity in an intergranular vortex. Such a structure is likely to form by advection of angular momentum towards the localized downflows by the familiar "bathtub effect". In fact, simulations of granular convection clearly show the formation of intense vortices (Nordlund, 1985a). On the observationally easier accesible mesogranular scale an example of such a vortical downflow has recently been observed (Brandt et al., 1988). Since the flux concentration cools its surroundings and thus enhances the converging downflows, magnetic structure and flow pattern can mutually stabilize each other: The strong thermal effects shape and stabilize the convective flow structure while this pattern, by means of advection of vorticity, stabilizes the magnetic element if its size is smaller than some critical value (Schüssler, 1984b). The observed deformation of granules around bright points (Muller et al., 1989) and the prolonged lifetime of the granular pattern in plage regions (Title et al., 1987) support this conjecture.

If for some reason, e.g. because of a major reorganisation of the pattern of convection, the supply of angular momentum becomes insufficient and the whirl decelerates, fluting 
instability sets in and the flux concentration is disrupted typically within the Alfvén transit time of less than a minute (Schüssler, 1986). The following evolution depends on the size of the fragments: If they are small enough (of the order of a few $\mathrm{km}$ ), magnetic diffusion becomes relevant and the fragments tend to disperse into weak fields which may go through another flux expulsion/convective collapse cycle. Larger fragments may survive long enough to be reassembled by the granular flows and fuse into new flux concentrations without the necessity of another convective collapse.

In this way, a dynamical view of small-scale photospheric magnetic fields emerges. At any given time, most of the flux is in magnetic elements, but the individual elements sooner or later split into fragments. Small fragments diffuse and, together with rising Uloops, contribute to a weak-field component which partially becomes reconcentrated by flux expulsion and convective collapse. Larger fragments (with a diameter $>10 \mathrm{~km}$, say) will rapidly heat up by radiation from the side leading to a decrease of the magnetic field strength. They are passive with respect to flows and may become severly distorted and inclined from the vertical direction before being assembled in integranular downdrafts to form new magnetic elements. All processes of splitting, diffusion, expulsion, collapse and accumulation of fragments operate in a timescale of minutes such that the majority of the flux at any given instant of time resides in the quasi-equilibrium strong-field form of magnetic elements.

\section{Conclusions}

The progress of our understanding of concentrated magnetic fields in the solar atmosphere achieved since the IAU-Symposium No. 102 in Zürich is considerable. The activity of research in this field has increased rapidly and a close interaction between theoretical and observational work has evolved. Comprehensive model calculations have been presented which reproduce the principal features of magnetic elements derived from observation without fine tuning of a large number of free parameters. These models begin to serve as tools for the diagnostics of solar magnetic structures by providing synthetic profiles of the full Stokes vector $(I, V, Q, U)$ of spectral lines which can be directly compared with observations (e.g. Grossmann-Doerth et al., 1989a). The concept of small-scale fields consisting mainly of ensembles of similar structures (magnetic elements) which may be described by basic flux tube or flux slab models embedded in a non-magnetic environment has turned out to be remarkably successful. A consistent picture of magnetic elements begins to emerge from sophisticated analysis of the spatially unresolved FTS data which have unsurpassed spectral quality (in terms of resolution, noise and wavelength range) and the basic physical effects which have been revealed by comprehensive model calculations and analytical studies of idealized problems. Let us try to summarize this picture:

Expulsion of magnetic flux by strong horizontal granular flows leads to magnetic flux concentrations in the intergranular lanes. Radiative cooling and the large superadiabaticity of the uppermost layers of the convection zone cause a strong local intensification of the magnetic field by way of a partial evacuation (convective collapse). A quasi-equlibrium evolves which is characterized by the absence of systematic internal flows and the balance of the magnetic pressure by an internal gas pressure deficit. The magnetic flux density decreases with height in a way well described by the slender flux tube approximation.

This equilibrium is stabilized against further collapse by a temperature deficit of the layers below optical depth unity in the magnetic structure due to the suppression of convective 
energy transport. Heating by lateral influx of radiation, reduced density and the strong temperature dependence of the continuum opacity cause the magnetic element to be much hotter than the quiet atmosphere at equal optical depth and to reach a temperature above $7000 \mathrm{~K}$ at $\tau_{c}=1$. Therefore, if observed with high spatial resolution near the center of the disc, the magnetic structure appears bright with a continuum intensity of about 1.5 times the value of the average Sun at $5000 \AA$. The "hot bottom and wall" of magnetic elements illuminate the upper layers of its atmosphere which becomes hotter than the environment even at equal geometrical depth. This contributes to the observed weakening of photospheric spectral lines.

The excess emission of magnetic elements is nearly compensated by an energy flux deficit in its environment such that only a small net excess flux is left. This cooling of the environment caused by lateral radiative energy flux into the magnetic element drives a thermal flow which supports, accelerates and stabilizes the granular downflows next to the magnetic structure. The external flows are responsible for the asymmetry of the observed Stokes $V$ profiles. Conservation of angular momentum leads to rapid rotation of these downflows which stabilizes the magnetic element with respect to the interchange/fluting instability.

The quasi-equilibrium state seems to be well represented by the FTS spectra. However, spectroscopic observations of individual magnetic structures with large spatial resolution are highly desirable in order to have an independent check of the methods which have been used to interpret the spatially unresolved FTS spectra. The challenging demand for high spatial and temporal resolution is compulsive for observational study of the formation and destruction processes, the dynamical interaction of the magnetic elements with convective flows, vortices and $p$-mode oscillations, the excitation and propagation of oscillations and waves within magnetic structures, and the interaction with other magnetic elements.

As far as theory is concerned, in contrast to some fashionable folklore existing and forthcoming numerical simulations do not make other approaches obsolete. The dynamics of motions and magnetic fields in the solar convection zone and atmosphere extends over huge ranges of temporal and spatial scales which in both case comprise more than ten decades. Since only a small part of these can be covered by a simulation, artificial boundaries have to be introduced, certain scales are ignored and others are includes only in a parametrized form. Such parametrizations can only be made in a sensible way if they are based on a sound understanding of processes which determine the properties of flows and fields on the scales which they attempt to describe.

The failure of 3D simulations to reproduce the differential rotation of the convection zone and the characteristics of the solar activity cycle has taught an important lesson: Unless we gain a better understanding of the effect of the small scales which then may lead to a reliable parametrization, comprehensive 3D simulations with low spatial resolution are potentially misleading. They are very helpful in drawing the attention to the relevant processes but these then have to be studied in detail in order to assess their general properties and consequences. This can be done by the analytical treatment of idealized problems and by simulations which gain spatial resolution at the expense of simplification, e.g. in dimensionality, of course keeping in mind that these approaches, due to their restrictions, might be misleading as well. So even in the case of solar granulation, a 3D time-dependent phenomenon par excellence, a 2D model (Steffen, these proceedings; Steffen et al., 1989) has apparently captured the basic physical situation (strong, localized, cool downflows and broad upflows, coupled by horizontal flows, advection and radiative transfer) and excellently reproduces the observed spectral and continuum features. Since such a model allows 
a much higher spatial grid resolution, it is well suited to study the effect of small scales and sharp gradients which may severly compromise 3D simulations. To qualify this work as "wrong" only because it is 2D (as it has been explicitely done in a summary talk at this conference) reveals a striking ignorance. In reality, comprehensive 3D simulations and idealized/simplified approaches are complimentary: The simulations help to identify the relevant processes and allow us a glimpse at phenomena which observationally are hidden behind a curtain of unsufficient spatial resolution or optically thick material. They can guide us in picking the relevant pieces of physics to study in detail without falling into the trap of oversimplified or prejudiced concepts. An understanding of the physics governing these processes, of their general properties and the validity of their description in the simulation can only come from a detailed study in the spirit of theoretical physics.

\section{References}

Akimov, L.A., Belkina, I.L., Dyatel, N.P.: 1982, Sov. Astron. 26, 334

Akimov, L.A., Belkina, I.L., Dyatel, N.P., Marchenko, G.P.: 1987, Sov. Astron. 31, 64

Anton, V.: 1989, Dissertation, Universität Göttingen

Auer, L.H., Heasley, J.N.: 1978, Astron. Astrophys. 64, 67

Bogdan, T.J., Knölker, M: 1989, Astrophys. J. 339, 579

Brandt, P., Scharmer, G.B., Ferguson, S., Shine, R.A., Tarbell, T.D., Title, A.M.: 1988, Nature 335, 238

Browning, P.K., Priest, E.R.: 1983, Astrophys. J. 266, 848

Chapman, G.A., Klabunde, D.P.: 1982, Astrophys. J. 261, 387

Degenhardt, D.: 1989, Astron. Astrophys., in press

Deinzer, W., Hensler, G., Schüssler, M., Weisshaar, E.:

1984a, Astron. Astrophys. 139, 426

Deinzer, W., Hensler, G., Schüssler, M., Weisshaar, E.:

1984b, Astron. Astrophys. 139, 435

Dunn, R.B., Zirker, J.B.: 1973, Solar Phys. 33, 281

Ferrari, A., Massaglia, S., Kalkofen, W., Rosner, R., Bodo, G.: 1985, Astrophys. J. 298, 181

Ferriz-Mas, A.: 1988, Phys. Fluids 31, 2583

Ferriz-Mas, A., Moreno-Insertis, F.: 1987, Astron. Astrophys. 179, 268

Ferriz Mas, A., Schüssler, M.: 1989, Geophys. Astrophys. Fluid Dyn., in press

Ferriz Mas, A., Schüssler, M., Anton, V.: 1989, Astron. Astrophys. 210, 425

Foukal, P., Fowler, L.: 1984, Astrophys. J. 281, 442

Foukal, P., Lean, J.: 1988, Astrophys. J. 328, 347

Galloway, D.J., Weiss, N.O.: 1981, Astrophys. J. 243, 945

Grossmann-Doerth, U., Knölker, M., Schüssler, M., Weisshaar, E.: 1989a, in R.J. Rutten and G. Severino (eds.): Solar and Stellar Granulation, Kluwer, Dordrecht, p. 481

Grossmann-Doerth, U., Schüssler, M., Solanki, S.K.: 1988, Astron. Astrophys. 206, L37

Grossmann-Doerth, U., Schüssler, M., Solanki, S.K.: 1989b, Astron. Astrophys., in press

Hasan, S.S.: 1983, see Stenflo (1983), p. 73

Hasan, S.S.: 1984, Astrophys. J. 285, 851

Hasan, S.S.: 1985, Astron. Astrophys. 143, 39

Hasan, S.S.: 1986, Mon. Not. Roy. Astron. Soc. 219, 357 
Hasan, S.S.: 1988, Astrophys. J. 332, 499

Hasan, S.S.: 1989, preprint

Hasan, S.S., Kneer, F.: 1986, Astron. Astrophys. 158, 288

Hasan, S.S., Schüssler, M.: 1985, Astron. Astrophys. 151, 69

Herbold, G., Ulmschneider, P., Spruit, H.C., Rosner, R.: 1985, Astron. Astrophys. 145, 157

Hirayama, T., Hamana, S., Mizugaki, K.: 1985, Solar Phys. 99, 43

Hollweg, J.V.: 1982, Astrophys. J. 257, 345

Howard, R.W., Stenflo, J.O.: 1972, Solar Phys. 22, 402

Hurlburt, N.E., Toomre, J.: 1988, Astrophys. J. 327, 920

Hurlburt, N.E., Toomre, J., Massaguer, J.M.: 1984, Astrophys. J. 282, 557

Illing, R.M.E., Landman, D.A., Mickey, D.L.: 1975, Astron. Astrophys. 41, 183

Kalkofen, W., Bodo, G., Massaglia, S., Rossi, P.: 1989, in R.J. Rutten and G. Severino (eds.): Solar and Stellar Granulation, Kluwer, Dordrecht, p. 571

Kalkofen, W., Rosner, R., Ferrari, A., Massaglia, S.: 1986, Astrophys. J. 304, 519

Kemp, J.C., Macek, J.H., Nehring, F.W.: 1984, Astrophys. J. 278, 863

Knölker, M., Schüssler, M.: 1988, Astron. Astrophys. 202, 275

Knölker, M., Schüssler, M., Weisshaar, E.: 1988, Astron. Astrophys. 194, 257

Landi Degl'Innocenti, E.: 1985, in H.U. Schmidt (ed.): Theoretical Problems in High Resolution Solar Physics, MPA 212, Max-Planck Institut für Astrophysik, München, p. 162

Lawrence, J.K., Chapman, G.A.: 1988, Astrophys. J. 335, 996

Livi, S.H.B., Wang, J., Martin, S.F.: 1985, Australian J. Phys. 38, 855

Martin, S.F., Livi, S.H.B., Wang, J.: 1985, Australian J. Phys. 38, 929

Massaglia, S., Bodo, G., Kalkofen, W., Rosner, R.: 1988, Astrophys. J. 333, 925

Massaglia, S., Bodo, G., Rossi, P.: 1989, Astron. Astrophys. 209, 399

Mehltretter, J.P.: 1974, Solar Phys. 38, 43

Meyer, F.: 1976, in R.-M. Bonnet, Ph. Delache (eds.): The Energy Balance and Hydrodynamics of the Solar Chromosphere and Corona, IAU-Colloq. No. 36, Nice, p. 111

Meyer, F., Schmidt, H.U., Simon, G.W., Weiss, N.O.: 1979, Astron. Astrophys. 76, 35

Meyer, F., Schmidt, H.U., Weiss, N.O.: 1977, Mon. Not. Roy. Astron. Soc. 179, 741

Montesinos, B., Thomas, J.H.: 1989, Astrophys. J. 337, 977

Muller, R.: 1975, Solar Phys. 45, 105

Muller, R.: 1983, Solar Phys. 85, 113

Muller, R., Roudier, Th.: 1984, Solar Phys. 94, 33

Muller, R., Roudier, Th., Hulot, J.C.: 1989, Solar Phys. 119, 229

Nordlund, A.: 1983, see Stenflo (1983), p. 79

Nordlund, $\AA .:$ 1984, in T.D. Guyenne and J.J. Hunt (eds.): The Hydromagnetics of the Sun, ESA SP-220, p. 37

Nordlund, A.: 1985a, in H.U. Schmidt (ed.): Theoretical Problems in High Resolution Solar Physics, MPA 212, Max-Planck Institut für Astrophysik, München, p. 1

Nordlund, A.: 1985b, in H.U. Schmidt (ed.): Theoretical Problems in High Resolution Solar Physics, MPA 212, Max-Planck Institut für Astrophysik, München, p. 101

Nordlund, $\AA .:$ 1986, in W. Deinzer et al. (eds.): Small Scale Magnetic Flux Concentrations in the Solar Photophere, Abh. Akad. d. Wiss. Göttingen No. 38, Vandenhoeck \& Ruprecht, Göttingen, p. 83

Osherovich, V., Flå, T., Chapman, G.A.: 1983, Astrophys. J. 268, 412 
Pantellini, F.G.E., Solanki, S.K., Stenflo, J.O.: 1988, Astron. Astrophys. 189, 263

Parker, E.N.: 1963, Astrophys. J. 138, 552

Parker, E.N.: 1975, Solar Phys. 40, 291

Parker, E.N.: 1978, Astrophys. J. 221, 368

Parker, E.N.: 1979, Cosmical Magnetic Fields, Clarendon, Oxford

Parker, E.N.: 1984, Astrophys. J. 283, 343

Pizzo, V.J.: 1986, Astrophys. J. 302, 785

Pneuman, G.W., Solanki, S.K., Stenflo, J.O.: 1986, Astron. Astrophys. 154, 231

Priest, E.R.: 1982, Solar Magneto-Hydrodynamics, Reidel, Dordrecht

Proctor, M.R.E., Weiss, N.O.: 1982, Rep. Progr. Phys. 45, 1317

Ribes, E., Rees, D.E., Fang, C.: 1985, Astrophys. J. 296, 268

Roberts, B. : 1976, Astrophys. J. 204, 268

Roberts, B., Webb, A.R.: 1978, Solar Phys. 56, 5

Rogerson, J.B.: 1961, Astrophys. J. 134, 331

Sanchez-Almeida, J., Collados, J., del Toro Iniesta, J.: 1988, Astron. Astrophys. 201, L37

Schatten, K.H., Mayr, H.G., Omidvar, K., Maier, E.: 1986, Astrophys. J. 311,460

Schmidt, H.U., Simon, G.W., Weiss, N.O.: 1985, Astron. Astrophys. 148, 191

Schmidt, H.U., Wegmann, R.: 1983, in B. Brosowski and E. Martensen (eds.): Dynamical Problems in Mathematical Physics, Lang, Frankfurt/Main, p. 137

Schüssler, M.: 1979, Astron. Astrophys. 71, 79

Schüssler, M.: 1984a, in T.D. Guyenne and J.J. Hunt (eds.): The Hydromagnetics of the Sun, ESA SP-220, p. 67

Schüssler, M.: 1984b, Astron. Astrophys. 140, 453

Schüssler, M.: 1986, in W. Deinzer et al. (eds.): Small Scale Magnetic Flux Concentrations in the Solar Photophere, Abh. Akad. d. Wiss. Göttingen No. 38, Vandenhoeck \& Ruprecht, Göttingen, p. 103

Schüssler, M.: 1987a, in E.-H. Schröter et al. (eds.): The Role of Fine-Scale Magnetic Fields on the Structure of the Solar Atmosphere, Cambridge University Press, p. 223

Schüssler, M.: 1987b, in B.R. Durney and S. Sofia (eds.): The Internal Solar Angular Velocity, Reidel, Dordrecht, p. 303

Schüssler, M., Solanki, S.K.: 1988, Astron. Astrophys. 192, 338

Simon, G.W., Weiss, N.O., Nye, A.: 1983, Solar Phys. 87, 65

Solanki, S.K.: 1986, Astron. Astrophys. 168, 311

Solanki, S.K.: 1989, Astron. Astrophys., in press

Solanki, S.K., Pahlke, K.-D.: 1988, Astron. Astrophys. 201, 143

Solanki, S.K., Stenflo, J.O.: 1984, Astron. Astrophys. 140, 185

Solanki, S.K., Stenflo, J.O.: 1986, Astron. Astrophys. 170, 120

Solov'ev, A.A.: 1984, Sov. Astron., 28, 54

Spruit, H.C.: 1976, Solar Phys. 50, 269

Spruit, H.C.: 1977, Solar Phys. 55, 3

Spruit, H.C.: 1979, Solar Phys. 61, 363

Spruit, H.C.: 1982, Astron. Astrophys. 108, 348

Spruit, H.C.: 1983, see Stenflo (1983), p. 41

Spruit, H.C., Roberts, B.: 1983, Nature 304, 401

Spruit, H.C., Schüssler, M., Solanki, S.K.: 1989, in A.N. Cox and W.C. Livingston (eds.):

The Solar Interior and Atmosphere, University of Arizona Press, Tucson, in press

Spruit, H.C., van Ballegooijen, A.A., Title, A.M.: 1987, Solar Phys. 110, 115 
Spruit, H.C., Zweibel, E.G.: 1979, Solar Phys. 62, 15

Steffen, M., Ludwig, H.-G., Krüß, A.: 1989, Astron. Astrophys. 213, 371

Steiner, O., Pizzo, V.J.: 1989, Astron. Astrophys. 211, 447

Steiner, O., Pneuman, G.W., Stenflo, J.O.: 1986, Astron. Astrophys. 170, 126

Stenflo, J.O. (ed.): 1983, Solar and Stellar Magnetic Fields: Origins and Coronal Effects, IAU-Symp. No. 102, Reidel, Dordrecht

Stenflo, J.O.: 1987, Solar Phys. 114, 1

Stenflo, J.O.: 1989, Astron. Astrophys. Rev. 1, 3

Stenflo, J.O., Harvey, J.W.: 1985, Solar Phys. 95, 99

Stenflo, J.O., Solanki, S.K., Harvey, J.W.: 1987, Astron. Astrophys. 171, 305

Sterling, A.C., Hollweg, J.V.: 1988, Astrophys. J. 327, 950

Suematsu, Y., Shibata, K., Nishikawa, T., Kitai, R.: 1982, Solar Phys. 75, 99

Thomas, J.H.: 1985, in H.U. Schmidt (ed.): Theoretical Problems in High Resolution Solar Physics, MPA 212, Max-Planck Institut für Astrophysik, München, p. 126

Thomas, J.H.: 1988, Astrophys. J. 337, 407

Title, A.M., Tarbell, T.D., Topka, K.P.: 1987, Astrophys. J. 317, 892

Title, A.M., Tarbell, T.D., Topka, K.P., Cauffman, D., Balke, C., Scharmer, G.: 1989, preprint

Tsinganos, K.C.: 1980, Astrophys. J. 239, 746

Unno, W., Ando, H.: 1979, Geophys. Astrophys. Fluid Dyn. 12, 107

Unno, W., Ribes, E.: 1979, Astron. Astrophys. 73, 314

Van Ballegooijen, A.A.: 1985, discussion remark in H.U. Schmidt (ed.): Theoretical Problems in High Resolution Solar Physics, MPA 212, Max-Planck Institut für Astrophysik, München, p. 177

Venkatakrishnan, P.: 1983, J. Astrophys. Astron. 4, 135

Venkatakrishnan, P.: 1985, J. Astrophys. Astron. 6, 21

Venkatakrishnan, P.: 1986, Nature 322, 156

Webb, A.R., Roberts, B.: 1978, Solar Phys. 59, 249

Weiss, N.O.: 1966, Proc. Roy. Soc. A293, 310

Weiss, N.O.: 1977, in E.A. Müller (ed.), Highlights of Astronomy 4, 241

Weiss, N.O.: 1981a, J. Fluid Mech. 108, 247

Weiss, N.O.: 1981b, J. Fluid Mech. 108, 273

Wiehr, E.: 1985, Astron. Astrophys. 149, 217

Wilson, P.R.: 1977, Astrophys. J. 214, 611

Zayer, I., Solanki, S.K., Stenflo, J.O.: 1989, Astron. Astrophys. 211, 463

Zwaan, C.: 1978, Solar Phys. 60, 213 